\title{
Nivel de Satisfacción e Intención de Repetir la Visita Turística. El Caso del Cantón Playas, en Ecuador
}

\author{
José R. Morales(1), Danny X. Arévalo(2), Carmen P. Padilla(2) y Miguel A. Bustamante ${ }^{(2,3) \star}$ \\ (1) Facultad de Ciencias Administrativas, Universidad de Guayaquil, Ciudadela Universitaria Salvador \\ Allende, Malecón del Salado entre Av. Fortunato Safadi (Av. Delta) y Av. Kennedy, Guayaquil, Ecuador. \\ (e-mail: roberto.moralesv@ug.edu.ec) \\ (2) Facultad de Ciencias Económicas y Empresariales, Universidad Católica Santiago de Guayaquil, \\ Guayaquil, Ecuador. Av. Carlos Julio Arosemena Km. 11/2 vía Daule, Guayaquil, Ecuador. \\ (e-mail: danny.arevalo@cu.ucsg.edu.ec; carmen.padilla@cu.ucsg.edu.ec \\ (3) Escuela de Ingeniería Comercial, Facultad de Economía y Negocios, Universidad de Talca, \\ Dos Norte 685, 3465548 Talca, Chile. (e-mail: mabu@utalca.cl) \\ * Autor a quien debe ser dirigida la correspondencia.
}

Recibido Mar. 14, 2017; Aceptado May. 16, 2017; Versión final Jul. 5, 2017, Publicado Feb. 2018

\begin{abstract}
Resumen
La presente investigación estudia la relación entre los diversos atributos que explican la satisfacción percibida por el turista y su influencia en la intención de repetir la visita turística al Cantón Playas, Ecuador. En el estudio se consideran algunas características socio-demográficas de los encuestados tales como estado civil, edad, ingreso mensual, ciudad de origen y el hecho de viajar solo o acompañado. Se aplicó una encuesta a una muestra final de 556 turistas en dos instancias de feriados locales. Se utilizó un análisis de regresión logística binaria como herramienta para determinar la probabilidad de repetir la visita turística. Los principales resultados del modelo evidenciaron que las variables socio-demográficas nivel de educación, ciudad de origen y viajar acompañado, así como atributos relacionados a la variedad de la oferta turística, precio, clima y los canales de distribución son estadísticamente significativos. En otras palabras, aquellos que respondieron positivamente a los cuatro atributos, tienen un alto grado de estudio, viven en zonas aledañas y viajan acompañados y tienen mayores probabilidades de repetir la visita al cantón Playas.
\end{abstract}

Palabras clave: satisfacción turística; repetición de visita; regresión logística binaria; atributos turísticos.

\section{Level of Satisfaction and Intention of Repeating the Touristic Visit. The Case of Canton Playas, in Ecuador}

\begin{abstract}
This work studies the relationship between various attributes that may explain the perceived satisfaction of tourists and their influence on the intention of repeating the visit to the Cantón Playas, Ecuador. The study considers some socio-demographic characteristics of the respondents such as marital status, age, monthly income, city of origin and whether they travel alone or accompanied. A survey was applied to a final sample of 556 tourists in two periods of local holidays. A binary logistic regression analysis was used as a tool to determine the likelihood of repeating the visit. The main results of the model showed that the sociodemographic variables level of education, city of origin and accompanied traveling, as well as attributes related to the variety of the touristic offer, price, climate and distribution channels are statistically significant. In other words, those who responded positively to the four attributes have a high level of education, live in surrounding areas and travel accompanied, are more likely to repeat the visit to Playas.
\end{abstract}

Keywords: tourist satisfaction; visit repetition; binary logistic regression; touristic attributes 


\section{INTRODUCCIÓN}

El turismo provee beneficios significativos para muchas economías especialmente aquellas en vías de desarrollo, generándoles ingresos adicionales de divisas y oportunidades de trabajo, entre otros beneficios (UNWTO, 1995). No obstante, este rápido desarrollo, genera efectos adversos como los producidos a los ecosistemas, que son visibles y se pueden encontrar en el uso excesivo de recursos por parte de los proveedores de servicios turísticos y otros se hacen evidentes en el poco respeto por la flora y fauna en los destinos turísticos (Scott, et al., 2012; Webster y Ivanov, 2014), razón por la cual el estudio del turismo resulta cada vez más relevante.

Es así como, los estudios de Sheldon y Dwyer (2010) señalan que es precisamente, durante las crisis económicas que este tipo de investigaciones se focalizan en los determinantes de la elección de un lugar de destino turístico y, en consecuencia también, la intención de repetir la visita es fundamental para comprender hasta qué punto, el gasto, se desvía hacia otros productos o destinos o bien, se acortan las estancias o se degradan los planes, percepción que, Hampson y McGoldrick (2013), complementan destacando el hecho de que los consumidores reaccionan muy rápidamente en tiempos de crisis, cambiando sus hábitos y muy difícilmente vuelven a los sitios ya visitados, razón por la cual, en un escenario de exigencia económica mundial, el éxito de los destinos turísticos depende de su capacidad de adaptación a las nuevas situaciones y, por lo tanto, resulta necesario y hasta imprescindible conocer, desde ya, las características y conducta de la demanda turística (Scott, et al., 2012).

\section{Revisión de la literatura}

El turismo es un término que presupone un concepto de fácil visualización y comprensión, aunque tiene un contenido más complejo de lo esperado. Para unos, es un fenómeno social y económico que abarca diversos grupos de interés, como son la industria del turismo, los turistas, las organizaciones gubernamentales y no gubernamentales y, las comunidades, que conforman los destinos turísticos (Song, et al., 2012). Por otra parte, los hallazgos del turismo se sintetizan en seis componentes principales, a decir: las denominadas atracciones reales, que incluyen las características naturales del paisaje y el clima como por ejemplo un destino de playa, así como atracciones artificiales, históricas, eventos culturales y deportivos , los componentes de servicio, que incluye aspectos como alojamiento, comida e instalaciones, que considera infraestructura, transporte, accesibilidad y compras, y el factor reputación, que abarca la imagen y percepción global del lugar de destino, además del elemento social, que indica cómo el destino es cuidado y apreciado por sus habitantes (Herington, et al., 2013) y, finalmente, el componente económico, relacionado con el atractivo económico del destino en relación con el valor inter-temporal por el dinero y el costo de la estadía, ratificando que, el turismo, es un concepto de alta dinámica y complejidad económica.

Es así como la demanda turística analiza variables explicativas socio-demográficas y económicas (Alén, 2016) tales como el nivel de ingreso de los turistas, edad, género, estatus profesional, los costos de destino, precios de sustitución, tipos de cambio y variables socio-demográficas que afectan el gasto turístico, mencionando entre éstas la composición del hogar, la edad, educación, ocupación y el ingreso, caracterizandolos como un determinantes significativos (García, et al., 2015). Complementariamente, otros estudios identifican que la edad y el sexo son factores determinantes en la intención de realizar una visita a un lugar de destino, a causa de no haber hallado diferencias significativas en las dimensiones situación económica o nivel educativo (Sung, et al. 2015). No obstante se reconoce que los atributos demográficos y socio-económicos por sí solos, no son suficientes para explicar los patrones de gasto turístico y la elección del lugar de destino (Luka, 2012).

A pesar de que las variables explicativas económicas desempeñan un papel importante en la decisión de viaje, diversos estudios han ampliado sus modelos para incluir variables no económicas de corte cualitativo que influyen en la elección de un destino en particular (Sung, et al., 2015). Estas variables incluyen experiencias previas, promociones de marketing, cercanía, variedad y calidad de productos y servicios turísticos, impacto del clima, inestabilidad política, entre otros (Prebensen, et al., 2010). Varios de estos atributos no sólo determinan la elección de una visita, sino que incrementan la satisfacción del turista con el lugar de destino (Herington et al., 2013; Rodríguez, et al., 2013). Adicionalmente, una de las motivaciones de viaje es la calidad de los productos turísticos, sin embargo, esto no tiene efecto significativo en el caso de visitantes repetitivos, pero tiene un efecto positivo para los nuevos visitantes.

En general, se aprecia que la imagen del destino es otro factor motivacional para la elección o repetición de la visita, entendida como la mejora o el deterioro de la imagen a priori del turista al finalizar su estadía (Stylos, et al., 2016) a lo que se agregan que, cuanto mejor sea la imagen de destino, mayor será la duración de la estadía y la probabilidad de repetir la visita. Sin embargo, se precisa una estructura clara que defina la relación que se produce entre la imagen del destino y la intención de visitar o volver a visitar un destino turístico (Tugcu, 2014). 
Otro elemento clave respecto del factor clima, es el tiempo de ocio, entendidos como determinantes de la elección de un destino de viaje, juicio que es compartido por otros estudios, confirmando que el clima no únicamente influye en la decisión de viajar a un destino en particular ( $\mathrm{Li}$, et al., 2017), sino que también es un factor clave que afecta la experiencia de viaje y, por consiguiente, determina la intención de repetir la visita en posteriores ocasiones. Es así como Wilson y Becken, 2011, encontraron además que la gente de países muy calurosos o muy fríos viajan más, al tiempo que, los países tropicales, son los que atraen a más turistas. En síntesis, el clima es un factor de empuje que impulsa la necesidad de movilización y atracción y, despierta el interés de ir hacia un destino turístico en particular (Saverimuttu y Varua, 2014).

La intención de regresar a un destino es también un indicador que ha sido ampliamente examinado debido, principalmente, a su relación con la satisfacción del turista con el lugar de destino (García, et al., 2015), especialmente a corto plazo. Incluso se afirma que la acción de repetir la visita es un concepto muy extendido en destinos turisticos maduros, principalmente los especializados en destinos de playa. Por otra parte, se argumenta que la intención de volver a visitar un determinado destino turístico es una recompensa al nivel de satisfacción percibido por el individuo durante su estadía en dicho destino (Assaker y Hallak, 2012), confirmando que la intención de repetir la visita se da en función del nivel de satisfacción, del valor percibido y del comportamiento pasado del individuo en sus elecciones turísticas.

Sintetizando los hallazgos, Alegre y Garau (2011) identificaron tres tipos de factores que pueden afectar la probabilidad de regresar a destinos de playa; los denominados factores básicos, que previenen al turista de sentirse insatisfecho; los factores de desempeño, entendidos como aquellos que aumentan los niveles de satisfacción de los turistas si se cumplen y lo contrario si no se cumplen; y finalmente, los factores de entusiasmo, que representa aquellos que incrementan la satisfacción si se cumplen, pero no reduce si esto no ocurre. Es así como se puede afirmar que a nivel de los estudios empíricos, existe una gran variedad de investigaciones que intentan explicar la probabilidad de repetición de la visita turística que datan de los años 90. La gran mayoría de estas investigaciones aprueban el uso de modelos binarios de elección discreta para determinar la significancia de los atributos de satisfacción, visitas previsas, características sociodemográficas y condiciones de viaje como variables exógenas (Alegre y Garau, 2011; Barros y Assaf, 2012), confirmando que, la intención de repetir la visita, puede ser analizada mediante métodos binarios, categorizando como afirmación o negación, la ejecución o no de dicha conducta.

Sin perjuicio de lo indicado, recientemente la literatura advierte de ciertos cambios demográficos que pueden afectar la industria del turismo en el mediano y largo plazo reorientando la estructura de oferta y demanda de turismo hacia el turismo senior, considerado como uno de los mayores mercados en crecimiento y más importante de la industria turística. La razón detrás de tal consideración radica en que los adultos mayores dan mayor importancia a los viajes en su tiempo de ocio durante la jubilación que las generaciones previas (Alén, et al., 2016). Otros estudios señalan al cambio climático como un factor que puede ser devastador para los destinos turísticos de verano (Saverimuttu y Varua, 2014), pudiendo ocasionar que algunos lugares turísticos luzcan menos atractivos pudiendo ser calificados como de entornos peligrosos, en tanto puedan acarrear efectos negativos y traer consigo el eventual declive del turismo y la generación de grandes pérdidas económicas para zonas determinadas (Scott et al., 2012).

Investigaciones sobre las interacciones entre turismo y cambio climático aparecieron por primera vez a mediados de los años 80, donde el primer foco de atención fue el impacto en destinos turísticos y sectores de alto riesgo como deportes de invierno y el turismo costero (Prebensen et al., 2010). No obstante, se observa en la actualidad un intenso debate sobre el cambio climático y sus efectos en la industria del turismo. Incluso se ha utilizado un índice de clima turístico que incluye múltiples variables climáticas relevantes para el turismo que examinan, de qué forma podría alterarse la distribución de los recursos climáticos para el turismo (García, et al., 2015). Los resultados de estos estudios revelan patrones geográficos consistentes con condiciones ideales para que la actividad turística se expanda en latitudes altas durante temporadas de verano, mientras que las condiciones se degraden en diversos y variados destinos subtropicales y tropicales de turismo.

En consecuencia, al verse alterados los factores climáticos que influyen en la elección del destino turístico, se proyecta que la competitividad mejorará en países como Canadá, Europa del Norte y Rusia, al tiempo que, disminuirá en países subtropicales y tropicales y podría existir además, un aumento de competitividad en destinos de playa en épocas de invierno. En este contexto, la adaptación a los impactos inducidos por el cambio climático se ha convertido en un asunto importante en la agenda de investigaciones de planificación del turismo (Dogru, et al., 2016; Foghagen, 2014). En este contexto, es que surge una nueva corriente denominada turismo sostenible (Kursah (2017) al puntualizar en el potencial del turismo sostenible representa la capacidad y oportunidad de elementos materiales e inmateriales para atraer a turistas a un destino que toma en consideración sus impactos socio-económicos y ambientales actuales y futuros, a la vez que satisface las necesidades de los visitantes, la industria, el medio ambiente y las comunidades de 
acogida. Sin embargo, la relación entre sostenibilidad y turismo es aún compleja (López-Sánchez y PulidoFernández, 2017), y existen pocos estudios enfocados en el concepto de turistas pro-sustentables así como aún escasos estudios acerca de la disposición de los turistas a pagar por dicha sostenibilidad, haciendo dificil identificar los factores claves que determinan el comportamiento de los turistas ante destinos sostenibles (Nejati, et al. 2015).

\section{Contextualización del turismo en Ecuador}

El turismo en el Ecuador ha sido tradicionalmente vinculado con destinos de playa. En estos destinos las temporadas de verano presuponen un clima tranquilo y agradable con sol y temperaturas altas (Prebensen et al., 2010), lo que se evidencia en los ingresos por turismo para los períodos comprendidos entre los años 2011 y 2015 que, en millones de dólares y de acuerdo con el último boletín del Ministerio de Turismo (MINTUR, 2017), muestra un aumento del consumo de turismo en el país. Este período también se destaca, por la campaña publicitaria ejecutada a nivel global denominada, "Al you need is Ecuador" como parte de la estrategia del gobierno para promocionar los distintos destinos turísticos que existen en el país.

Durante los tres primeros períodos del análisis (2011-2013), el turismo ocupó el cuarto lugar dentro de la categoría de ingresos por turismo y exportaciones según producto principal, ubicación que hace referencia al puesto que ocupa una actividad en función del ingreso de divisas, únicamente por debajo de productos como el banano, camarón, manufactura de metales y flores naturales. Para los años 2014 y 2015, el sector del turismo muestra progresos sustanciales ocupando el tercer lugar de prioridad relativa de los sectores económicos más aportantes al ingreso nacional.

El Cantón Playas, Ecuador, que hace parte de la provincia del Guayas con 42.935 habitantes y cuenta con una extensa playa de aproximadamente $14 \mathrm{~km}$ de longitud (INEC, 2017), está localizado en el suroeste del Ecuador, noroeste de América Latina, a orillas del océano Pacífico, a unos 96 kilómetros de la ciudad de Guayaquil. En la actualidad, Playas se ha convertido en el principal y único balneario con acceso al mar dentro de la provincia más poblada del Ecuador y, por ende, está llamado a convertirse en un centro turístico por el potencial que posee. Entre sus principales atractivos turísticos se encuentran paisajes naturales, hoteles, restaurantes, centros de entretenimiento, entre otros.

A la luz de los factores mencionados es que surge el objetivo principal de este estudio que consiste en estudiar la relación entre los diversos atributos que explican la satisfacción percibida del turista y su influencia en la intención de repetir la visita turística, al Cantón Playas, Ecuador, considerando algunas características socio - demográficas tales como estado civil, edad, ingreso mensual, ciudad de origen y el viajar solo o acompañado.

\section{METODOLOGÍA}

La metodología del presente estudio es mixta, es decir de tipo cualitativa y cuantitativa, de corte transversal con fuentes de datos primarios y de naturaleza correlacional - causal y explicativa (Hernández et al., 2006; Eyssautier, 2006). Para el desarrollo del estudio se utilizó el instrumento de Alegre y Garau (2011) que consta de 24 atributos que determinan la satisfacción de los turistas con destinos de balnearios y playas y, dado que los mismos autores reconocieron presencia de problemas de multicolinealidad entre algunos de sus atributos, se realizó una evaluación de expertos como medida para seleccionar aquellos atributos más afines para el estudio.

Dentro de la fase cualitativa se optó por la elección del método Delphi, a fin lograr un consenso con base en la discusión entre expertos (Hernández et al., 2006), en este caso, destinada a modificar el diseño del cuestionarios original. El panel de expertos se llevó a cabo la tercera semana del mes de julio de 2016 y estuvo conformado por representantes tanto de la Alcaldía como de la Cámara de Turismo de Playas y tres gerentes de importantes hoteles del sector. Complementariamente, se recogieron comentarios de expertos académicos y se llegó al consenso de utilizar 9 atributos considerados indispensables para examinar la satisfacción de los turistas y su intención de volver a visitar el lugar.

El resultado final fue un cuestionario refinado con 10 preguntas, 9 de las cuales corresponden al nivel de satisfacción percibido en los nueve atributos medido a través de una escala Likert de cinco puntos, donde uno es totalmente en desacuerdo y cinco totalmente de acuerdo. La última pregunta es de tipo dicotómica y mide la intención de repetir la visita al Cantón Playas. Se agregaron además otras preguntas de corte sociodemográfico, como sexo, edad, nivel de educación, estado civil, entre otros. Los nueve atributos se resumen en la Tabla 1. 
Tabla 1: Atributos seleccionados para el presente estudio

\begin{tabular}{|c|c|}
\hline Atributo & Descripción \\
\hline $\begin{array}{l}\text { Variedad de } \\
\text { productos turísticos }\end{array}$ & $\begin{array}{l}\text { Se refiere a la amplitud de ofertas que existen en el cantón tales como visitar museos, hostales, } \\
\text { deportes acuáticos, playas, discotecas, etc. }\end{array}$ \\
\hline Seguridad & $\begin{array}{l}\text { Trata de una variable básica de confort de los visitantes, especialmente por concurrir a un sitio } \\
\text { ajeno a su habitualidad }\end{array}$ \\
\hline Precios económicos & Como percepción de equidad al poder acceder a valoraciones justas de servicios \\
\hline $\begin{array}{l}\text { Calidad de los } \\
\text { productos turísticos }\end{array}$ & Calidad medida en términos de acceso, confort y calidez de servicio, entre otros. \\
\hline $\begin{array}{l}\text { Infraestructura } \\
\text { hotelera }\end{array}$ & Como expresión de solvencia y estabilidad de la oferta turística que se les presenta \\
\hline Cercanía & $\begin{array}{l}\text { Considerada una característica muy valiosa para el visitante en términos del esfuerzo que mide } \\
\text { el tiempo de movilización entre su lugar de origen y el destino }\end{array}$ \\
\hline Publicidad & En términos de claridad y la veracidad de la información recibida \\
\hline $\begin{array}{l}\text { Canales de } \\
\text { distribución }\end{array}$ & $\begin{array}{l}\text { Refiere al fácil acceso a información mediante los medios de difusión que los ofertantes } \\
\text { turísticos emplean para llegar a los visitantes }\end{array}$ \\
\hline Clima & $\begin{array}{l}\text { Uno de los atributos diferenciadores valorados por los turistas referente a la percepción } \\
\text { deseada del ambiente }\end{array}$ \\
\hline
\end{tabular}

Debido a que los ítems del cuestionario fueron adaptados de una investigación previa y se escogieron únicamente nueve para satisfacer las necesidades de la investigación, se realizó una prueba piloto para asegurar la confiabilidad de las escalas considerando que el valor límite para aceptar fue de un alfa de Cronbach de 0,7 , que garantice consistencia interna. La prueba piloto se realizó durante la primera semana de agosto de 2016, encuestándose a 41 turistas en el malecón de Playas. Los datos fueron tabulados y analizados con el software estadístico SPSS 22 y se obtuvo resultados satisfactorios, logrando un índice de fiabilidad que arrojó un coeficiente Alfa de Cronbach de 0,895 que demuestra que el instrumento posee confiabilidad alta.

Verificada la confiabilidad del instrumento, se realizó un muestreo aleatorio simple para encuestar personas en las playas y en otros sectores de concurrencia masiva como malecón y reserva natural (Hernández et al., 2006), logrando totalizar 600 turistas de los cuales se obtuvieron 556 cuestionarios validos una vez eliminados aquellos con inconsistencias, datos atípicos o preguntas sin respuestas. En el trabajo de campo, se consideró un criterio de inclusión - exclusión, solicitándole al encuestado indicar si se alojaría en el lugar o indicara si sólo estaría de visita ese mismo día. De este modo el estudio consideró únicamente aquellas personas que habían pasado o tenían pensado pasar un día o más en el destino. Se excluyeron en consecuencia, los excursionistas que manifestaron una permanencia solo por el día sin pernoctar en el lugar, evitando así problemas de sesgo en los resultados.

La recolección de información se dio en dos instancias. En una primera instancia se encuestó a turistas que concurrieron a Playas el feriado del 10 de agosto de 2016, transferido a los días 12, 13 y 14 agosto del mismo año, dando como resultado un número de 384 cuestionarios válidos; en una segunda instancia, se encuestaron a turistas que visitaron Playas durante el feriado de carnaval los días 25, 26 y 27 de febrero de 2017, obteniendo un total de 172 cuestionarios válidos, totalizando una muestra de 556 unidades.

La técnica estadística considerada para el estudio fue el análisis de regresión logística binaria, se definió como variable dependiente y endógena del modelo, la intención de repetir la visita turística al Cantón Playas en la provincia del Guayas, misma que fue configurada como una variable dicotómica (Hair, et al., 2011), considerando que, de acuerdo con el método, una respuesta positiva representa la intención de visitar nuevamente el destino turístico y una respuesta negativa que no existe tal intención.

Se aplicaron adicionalmente, otros mecanismos de estadística descriptiva y comparación de medias para mayor entendimiento de las variables seleccionadas y determinar diferencias significativas de las respuestas obtenidas en las dos instancias de la muestra, a fin de verificar los hallazgos y aceptar 0 rechazar las hipótesis. La Tabla 2, muestra las hipótesis de investigación del presente estudio. 
Tabla 2: Planteamiento de las hipótesis de investigación

\begin{tabular}{|c|c|}
\hline Hipótesis & Descripción \\
\hline $\mathrm{H}_{1}$ & $\begin{array}{l}\text { Los nueve atributos seleccionados influyen significativamente en la intención de repetir la visita turística } \\
\text { en el cantón Playas }\end{array}$ \\
\hline $\mathrm{H}_{1 \mathrm{a}}$ & $\begin{array}{l}\text { La variedad de productos turísticos influye significativamente en la intención de repetir la visita turística en } \\
\text { el cantón Playas }\end{array}$ \\
\hline $\mathrm{H}_{1 \mathrm{~b}}$ & La seguridad influye significativamente en la intención de repetir la visita turística en el cantón Playas \\
\hline $\mathrm{H}_{1 \mathrm{c}}$ & El precio influye significativamente en la intención de repetir la visita turística en el cantón Playas \\
\hline $\mathrm{H}_{1 \mathrm{~d}}$ & La calidad influye significativamente en la intención de repetir la visita turística en el cantón Playas \\
\hline $\mathrm{H}_{1 \mathrm{e}}$ & $\begin{array}{l}\text { La infraestructura turística influye significativamente en la intención de repetir la visita turística en el } \\
\text { cantón Playas }\end{array}$ \\
\hline $\mathrm{H}_{1 f}$ & La cercanía influye significativamente en la intención de repetir la visita turística en el cantón Playas \\
\hline $\mathrm{H}_{1 \mathrm{~g}}$ & La publicidad influye significativamente en la intención de repetir la visita turística en el cantón Playas \\
\hline $\mathrm{H}_{1 \mathrm{~h}}$ & $\begin{array}{l}\text { Los canales de distribución influyen significativamente en la intención de repetir la visita turística en el } \\
\text { cantón Playas }\end{array}$ \\
\hline $\mathrm{H}_{1 \mathrm{i}}$ & El clima influye significativamente en la intención de repetir la visita turística en el cantón Playas \\
\hline
\end{tabular}

\section{RESULTADOS}

Los resultados presentan un análisis de estadística descriptiva de las principales variables sociodemográficas y los atributos que inciden en la intención de repetir la visita en el destino estudiado, las comparaciones de medias para examinar diferencias significativas existentes entre instancias de recogida de datos y un modelo de regresión logística binaria para determinar la incidencia de los atributos seleccionados en el estudio en la probabilidad de retorno de los turistas a Playas.

\section{Estadística descriptiva}

La tabla 3 muestra los resultados de estadística descriptiva para las variables socio-demográficas. Para las variables continuas se presenta la media mientras que para las variables categóricas se presenta la frecuencia.

Tabla 3: Estadística descriptiva de variables socio-demográficas

\begin{tabular}{|c|c|c|c|c|c|}
\hline Variable & Definición & $\begin{array}{l}\text { Media o } \\
\text { frecuencia }\end{array}$ & Variable & Definición & $\begin{array}{l}\text { Media o } \\
\text { frecuencia }\end{array}$ \\
\hline Edad & En años (rango: 18-74) & 34,99 & $\begin{array}{l}\text { Ciudad de } \\
\text { origen }\end{array}$ & Guayaquil & $38,49 \%$ \\
\hline \multirow[t]{7}{*}{ Educación } & $1=$ Primaria incompleta & $0,54 \%$ & & Cuenca & $11,33 \%$ \\
\hline & $2=$ Primaria completa & $3,60 \%$ & & Quito & $5,58 \%$ \\
\hline & $3=$ Secundaria incompleta & $8,45 \%$ & & El Triunfo & $6,12 \%$ \\
\hline & $4=$ Secundaria completa & $21,04 \%$ & & Bucay & $2,34 \%$ \\
\hline & $5=$ Universitaria incompleta & $25,54 \%$ & & Duran & $8,63 \%$ \\
\hline & $6=$ Universitaria completa & $33,81 \%$ & & Manta & $1,98 \%$ \\
\hline & 7 = Estudios de posgrado & $7,01 \%$ & & Daule & $3,06 \%$ \\
\hline Ingreso mensual & En dólares americanos & 894,13 & & Babahoyo & $1,80 \%$ \\
\hline \multirow[t]{5}{*}{ Estado civil } & 1 = Casado & $46,22 \%$ & \multirow{5}{*}{$\begin{array}{l}\text { Ciudad de } \\
\text { origen } 2\end{array}$} & Balzar & $1,44 \%$ \\
\hline & 2 = Soltero & $53,06 \%$ & & Extranjeros & $1,98 \%$ \\
\hline & $3=$ Viudo & $0,36 \%$ & & Otras & $17,27 \%$ \\
\hline & 4 = Divorciado & $0,36 \%$ & & $\begin{array}{l}1=\text { Ciudades a menos } \\
\text { de } 4 \text { horas conduciendo }\end{array}$ & $63,85 \%$ \\
\hline & & & & $\begin{array}{l}2 \text { = Ciudades a más de } \\
4 \text { horas conduciendo }\end{array}$ & $36,15 \%$ \\
\hline \multicolumn{2}{|c|}{ Viaja acompañado } & $71,90 \%$ & & & \\
\hline \multicolumn{2}{|l|}{ Intención de volver } & $74,30 \%$ & & $\mathrm{~N}$ & 556 \\
\hline
\end{tabular}


Se destaca que la edad media de los encuestados que visitan el cantón Playas es de 35 años. El nivel de educación evidencia que la mayor parte de los turistas encuestados poseen estudios universitarios terminados $(33,81 \%)$, seguido por personas con educación universitaria incompleta $(25,54 \%)$. Por otro lado el ingreso promedio mensual de los encuestados es de USD 894,13 y, respecto al estado civil, un $53,06 \%$ de los encuestados respondieron estar solteros, seguido por un $46,22 \%$ de encuestados afirmando estar casados. Otra variable de interés es la ciudad de origen de los turistas. Los resultados destacan que la mayoría de visitantes del cantón Playas provienen de la ciudad de Guayaquil (38\%), situada a una hora de recorrido en automóvil. Esta variable fue recodificada para ser considerada en el modelo como variable ordinal de acuerdo a la distancia de cada ciudad medida en horas en automóvil. En resumen, la mayoría de los visitantes provienen de Guayaquil y sectores aledaños con un $63,85 \%$, mientras que se registra pocas visitas de otras ciudades importantes como Quito, Cuenca e inclusive extranjeros, con un 36,15\%. Por último, el 71,90\% de los encuestados indicaron que suelen viajar acompañados. Por último, la variable endógena binaria del modelo que representa la probabilidad de retorno al destino evidencia que el $74,30 \%$ de los turistas están satisfecho con su visita al cantón de Playas y tiene la intención de regresar a visitar el lugar.

La tabla 4 expone los resultados de estadística descriptiva para los nueve atributos seleccionados en el estudio que representan la satisfacción del turista con el lugar de destino. Se evidencia que el clima representa el atributo de mayor interés en los turistas. Los resultados exhiben que el $49,46 \%$ de los turistas calificó como totalmente de acuerdo su grado de satisfacción con el clima. A este atributo le sigue el de cercanía. El 36,66\% de los encuestados indicaron estar parcialmente de acuerdo mientras que el 31,47\% indicó estar totalmente de acuerdo. Otros atributos concentraron sus respuestas en la cuarta categoría parcialmente de acuerdo, como es el caso de la variedad y calidad de productos turísticos y la seguridad. Los turistas encuestados concuerdan que el cantón ofrece una importante variedad y calidad de productos turísticos tales como hospedaje, restaurantes, bares, actividades acuáticas, entre otros.

Los atributos infraestructura turística, precio y publicidad concentran las respuestas en una categoría neutra con el $35,61 \%, 44,42 \%$ y $39,57 \%$ respectivamente. Esto indica que estos atributos no elevan en grandes proporciones la satisfacción con el lugar de destino, pero tampoco la disminuyen. En otras palabras, los turistas no están ni de acuerdo ni en desacuerdo con la infraestructura turística actual del cantón, ni con los precios de sus diversos productos turísticos, así como con la publicidad existente para atraer más visitantes a la zona. Finalmente, los encuestados respondieron estar en mayor medida parcialmente en desacuerdo con el atributo canales de distribución. Esto quiere decir que los medios por los cuales el cantón es publicitado no es el adecuado y no ofrecen facilidades en el acceso a la información para planificar una visita más placentera, aprovechando al máximo todos los beneficios y comodidades que el destino oferta.

Tabla 4: Estadística descriptiva de los atributos de satisfacción con el lugar de destino

\begin{tabular}{lccccc}
\hline \multicolumn{1}{c}{ Atributos } & $\begin{array}{c}\text { Totalmente en } \\
\text { desacuerdo }\end{array}$ & $\begin{array}{c}\text { Parcialmente } \\
\text { de acuerdo }\end{array}$ & Neutral & $\begin{array}{c}\text { Parcialmente } \\
\text { de acuerdo }\end{array}$ & $\begin{array}{c}\text { Totalmente } \\
\text { de acuerdo }\end{array}$ \\
\hline Variedad de productos turísticos & 0,72 & $(2)$ & $(3)$ & $(4)$ & $(5)$ \\
Seguridad & 0,72 & 6,86 & 32,37 & 55,94 & 6,12 \\
Precios & 0,36 & 5,58 & 27,34 & 42,63 & 22,84 \\
Calidad de productos turísticos & - & 6,29 & 33,45 & 43,71 & 13,67 \\
Infraestructura turística & 7,73 & 18,53 & 35,61 & 29,14 & 8,99 \\
Cercanía & 0,90 & 7,19 & 24,10 & 36,33 & 31,47 \\
Publicidad & 8,09 & 19,96 & 39,57 & 25,54 & 6,83 \\
Canales de distribución & 6,12 & 33,09 & 22,84 & 26,80 & 11,15 \\
Clima & 0,18 & 3,06 & 11,51 & 35,79 & 49,46 \\
\hline
\end{tabular}

\section{Diferencia de medias}

El análisis de diferencia de medias, realizado para comprobar si las respuestas de las diferentes instancias en las cuales se recogió los datos del estudio varían o no estadísticamente y, puedan o no ser consideradas como una misma población se muestra en la tabla 5. 
Tabla 5: Prueba de Kruskal-Wallis de diferencia de medias

\begin{tabular}{|c|c|c|c|c|c|c|c|c|c|}
\hline \multicolumn{2}{|c|}{$\begin{array}{c}\text { Variedad de productos } \\
\text { turísticos }\end{array}$} & \multirow{2}{*}{$\begin{array}{c}\text { Seguridad } \\
1,769\end{array}$} & \multirow{2}{*}{$\begin{array}{c}\text { Precio } \\
, 092\end{array}$} & \multirow{2}{*}{$\begin{array}{c}\text { Calidad } \\
251\end{array}$} & \multirow{2}{*}{$\begin{array}{c}\text { Infraestructur } \\
\text { a turística }\end{array}$} & \multirow{2}{*}{$\begin{array}{c}\text { Cercanía } \\
\text {,442 }\end{array}$} & \multirow{2}{*}{$\begin{array}{c}\text { Publicidad } \\
, 176\end{array}$} & \multirow{2}{*}{$\begin{array}{c}\begin{array}{c}\text { Canales de } \\
\text { distribución }\end{array} \\
, 090\end{array}$} & \multirow{2}{*}{$\begin{array}{l}\text { Clima } \\
, 019\end{array}$} \\
\hline Chi-cuadrado & 1,723 & & & & & & & & \\
\hline gl & 1 & 1 & 1 & 1 & 1 & 1 & 1 & 1 & 1 \\
\hline Sig. asintótica & , 189 & ,183 & ,761 & ,616 & ,711 &, 506 & 675 & ,764 & ,890 \\
\hline
\end{tabular}

Este análisis se realizó mediante la prueba de Kruskall-Wallis, mayormente utilizada en variables categóricas, para los nueve atributos de satisfacción con el lugar de destino. En consecuencia, debido a que todos los valores son mayores al nivel de significancia de 0,05 , se concluye que no existe diferencias significativas entre ambas instancias del estudio y, por consiguiente, los datos pueden ser estudiados de manera integrada como una sola muestra (Lind, et al., 2012).

\section{Determinando la intención de retorno}

La Tabla 6 resume los resultados de la regresión logística binaria de todas las variables significativas del estudio, considerando variables significativas a un nivel ${ }^{* * *} p<0,01,{ }^{* *} p<0,05,{ }^{*} p<0,1$ basado en estadístico de Wald. En resumen, se observa que cuatro de los nueve atributos que explican la satisfacción con la visita del destino son significativas. Por otro lado, sólo tres variables socio-demográficas son significativas con respecto a la variable endógena intención de repetir la visita.

En general, las tres variables socio-demográficas ciudad de origen, viajar acompañado y nivel de educación son predictores importantes en la intención de repetir la visita a un nivel de significancia $p$ de 0,01 . De estas tres, la variable que más poder de predicción posee es el hecho de viajar acompañado. Esto indica que los turistas que viajan acompañados de sus parejas, familia o amigos, presentan niveles de satisfacción altos con el lugar de destino y que, siempre que el lugar de destino sea de agrado común, eleva la probabilidad de retorno en 2,23 puntos. A esta variable le sigue la ciudad de origen. El tiempo de arribo al cantón, que hace referencia al tiempo que los turistas domiciliados en las distintas ciudades del Ecuador recorren hasta llegar al cantón, presenta un impacto positivo y significativo. Esto quiere decir que, a medida que la persona se encuentre en una ciudad a menor distancia del balneario, encuentra mayor probabilidad en la repetición de la visita.

Tabla 6: Regresión logística binomial con las variables significativas

\begin{tabular}{|c|c|c|c|c|}
\hline Variables & $B^{a}$ & Error estándar & Wald & $\operatorname{Exp}(B)$ \\
\hline Variedad productos turísticos & $0,2648^{*}$ & 153 & 3,012 & 1,303 \\
\hline Precios & $0,2974^{* *}$ & 130 & 5,204 & ,743 \\
\hline Canales distribución & $0,2093^{\star *}$ & ,096 & 4,758 & 1,233 \\
\hline Clima & $0,2506^{* *}$ &, 126 & 3,962 & 1,285 \\
\hline Ciudad de origen 2 & $0,6338^{* * *}$ & ,230 & 7,628 & 1,885 \\
\hline Viajar acompañado & $0,8021^{\star * *}$ & ,220 & 13,278 & 2,230 \\
\hline Nivel educación & $0,2925^{\star \star *}$ & 111 & 7,006 & 1,340 \\
\hline Prueba Hosmer y Lemeshow & $\chi^{2}=11,492$ & Sig. $=0,175$ & & \\
\hline $\mathrm{R}^{2}$ de Cox y Snell & 0,103 & & & \\
\hline $\mathrm{R}^{2}$ de Nagelkerke & 0,151 & & & \\
\hline \multirow{2}{*}{ Análisis COR } & Área & D.E. & Sig. & Límites \\
\hline & ,702 & ,025 & $p<0,01$ & {$[0,653-0,752]$} \\
\hline
\end{tabular}

Se observa que cuatro de las nueve hipótesis planteadas han sido comprobadas en el presente estudio $\left(\mathrm{H}_{1 \mathrm{a}}, \mathrm{H}_{1 \mathrm{c}}, \mathrm{H}_{1 \mathrm{~h}}\right.$ y $\left.\mathrm{H}_{1 \mathrm{i}}\right)$. Analizando detenidamente los atributos que aportan a la satisfacción del turista con el lugar de destino, se encuentra que las variables precio, canales de distribución y clima presentan 
significancia estadística positiva a un nivel $p$ de 0,05 , mientras que variedad de productos turísticos presenta significancia estadística a un nivel $p$ de 0,1 . El atributo que mayor poder de predicción exhibe es la oferta de variedad de productos turísticos. Se puede afirmar entonces que la variedad de los productos turísticos tales como parques, paisajes naturales, playas paradisíacas, entre otros, genera mayor satisfacción y, por consiguiente, un efecto positivo respecto a la intención de repetir la visita al cantón (Alegre et al., 2011). En síntesis, la probabilidad de repetición aumenta en 1,303 puntos a medida que existe un mayor portafolio de productos y servicios turísticos que el destino oferta.

De las variables significativas a un nivel de confianza de 95\%, el clima representa el atributo con mejor capacidad de predicción. Esto implica que los turistas repiten la visita cuando el destino ofrece un clima agradable. Estos resultados concuerdan con estudios previos que indican que el clima es una variable significativa en la predicción de la satisfacción con el lugar turístico y motivante de la repetición de la visita (Saverimuttu y Varua, 2014; Scott et al., 2012) y que, en la actualidad, los destinos de playas más visitados son aquellos que mantienen un clima agradable y satisfactorio, dado el deterioro constante del medio ambiente (Dogru et al., 2016).

El atributo canales de distribución es otra variable significativa del estudio. Los resultados evidencian que el fácil acceso a la información por las vías adecuadas permite al visitante una mejor planificación de su estadía, a la par de motivar la repetición de la visita al destino. Estos resultados guardan relación con los hallazgos de Lai y Vinh (2013), quienes sostienen que la eficacia promocional del turismo está vinculada con el servicio y la satisfacción turística. Por otra parte, el precio resultó ser un tema de mucho interés al momento de decidir un destino a visitar. Los resultados evidencian que en la medida que el precio satisface las expectativas del turista, eleva la probabilidad de repetir la visita. Esto es corroborado por la literatura acerca de los gastos del turista en destinos de playa (Alegre et al., 2011). No obstante, este atributo presenta la capacidad de predicción más baja del modelo. Esto puede darse por la creciente tendencia de la práctica del turismo sostenible. Este término de sostenibilidad, guarda relación con el equilibrio entre la satisfacción de la necesidad de los grupos de interés y el precio que el turista está dispuesto a pagar por los servicios recibidos. Estudios señalan que los destinos de playa tendrían que afrontar cambios para ser competitivos dados los actuales y futuros problemas de tipo demográfico y climático que se podrían enfrentar (López-Sánchez y Pulido-Fernández, 2017).

La variable calidad no resultó ser suficientemente representativa en el presente estudio, puesto que, como era de esperar, las personas provenientes de economías en vías de desarrollo, son más sensibles a los factores monetarios (Webster y Ivanov, 2014) por lo que, evidentemente, a la hora de decidir, priorizan el precio sobre la calidad. Sin embargo, a medida que se expanda la práctica de turismo sostenible, la conciencia por la calidad se estima podría aumentar, como lo indican estudios recientes (Kursah, 2017; López-Sánchez y Pulido-Fernández, 2017).

Otras variables como seguridad, infraestructura, cercanía y publicidad no resultaron ser significativas en el presente estudio. El atributo cercanía de igual modo arrojó valores no significativos, sin perjuicio que, al parecer, este atributo guarda cierta relación con la variable ciudad de origen 2, existiendo una clara diferencia. La primera trata del esfuerzo medido en tiempo para acceder al lugar de destino, mientras que el segundo está más enfocado hacia el lugar de donde proviene el individuo. Los datos iniciales arrojaron que la mayor parte de los encuestados provienen de Guayaquil y otras ciudades de la provincia. En consecuencia, este antecedente establece que los habitantes de zonas aledañas prefieren visitar el cantón Playas con más frecuencia que aquellos residentes provenientes de otras provincias del Ecuador; no obstante ello, los resultados evidencian que la cercanía no es un impedimento al momento de seleccionar un destino a visitar. Por último, la publicidad no arrojó valores significativos como si presentó el atributo canales de distribución, dejando de manifiesto que no es la información per sé lo que motiva al usuario repetir la visita, sino cuán fácil es el acceso a la información que aumente la probabilidad de decisión por elegir un destino por una siguiente ocasión.

En general, se realizaron diversas pruebas para determinar la capacidad de ajuste del modelo (Hair et al., 2011). La prueba de Hosmer y Lemeshow permite evaluar la bondad de ajuste de un modelo de regresión logística. Si el ajuste es bueno, un valor alto de la probabilidad predicha $p$ se asociará con el resultado 1 de la variable binomial. Lo que se desea es que no exista significación estadística en esta prueba. Dado que el valor $p>0,05$, se afirma que el modelo presenta un buen ajuste entre los datos observados versus los predichos. El R ${ }^{2}$ de Nagelkerke es de 0,151 indicando que el modelo explica aproximadamente el $15 \%$ de la variabilidad de la variable endógena.

A pesar de que la prueba de Hosmer y Lemeshow evalúa un aspecto de validez y el $\mathrm{R}^{2}$ de $\mathrm{Nagelkerke}$ adopta valores bajos debido a la poca variabilidad de los modelos binarios, fue necesaria una prueba de discriminación del modelo, es decir, del grado en que este distingue entre individuos que repiten la visita al 
lugar de destino y los que no. Para ello se efectuó el análisis ROC, prueba construida para la probabilidad predicha. El área bajo la curva representa el grado en que el modelo clasifica adecuadamente los individuos. La literatura sugiere que valores mayores a 0,6 son satisfactorios (Hair et al., 2011; Lind et al., 2012), en consecuencia, dado que el área bajo la curva ROC fue de $70,2 \%$, la sensitividad fue de $63 \%$ y la especificidad de $36,4 \%$, implicando que quienes respondieron positivamente a los cuatro atributos, tienen un alto grado de estudio, viven en zonas aledañas y viajan acompañado, tienen mayores probabilidades de repetir la visita al lugar de destino.

\section{CONCLUSIONES}

Las variables socio-demográficas nivel de educación, ciudad de origen y preferencia por viajar acompañado influyen en la elección de un destino turístico y se confirma que los atributos variedad de productos, precios, canales de distribución y clima son predictores en la intención de repetir la visita al lugar de destino. Además, la mayoría de los encuestados mostraron un alto nivel de satisfacción con la variable cercanía, sin embargo, no resultó significativa, evidenciando que la distancia no es impedimento al momento de elegir el lugar de destino.

Algunos determinantes identificados para la elección y repetición al lugar de destino turístico resultaron ser el clima como elemento de satisfacción del consumidor, la sensibilidad al precio del consumidor para la elección del destino, la disposición de productos y servicios turísticos y la eficacia de los canales de distribución para el acceso a la información.

Los hallazgos del estudio presentan diversas implicaciones tanto para los responsables de las políticas públicas como para las empresas. Por un lado el lugar de destino y el clima de la zona, la variedad de productos turísticos y del precio como fuente de satisfacción; además de dimensionar el efecto de las variables socio-demográficas significativas que explican la satisfacción y conducta de repetición de la visita. Complementariamente, parece razonable considerar los efectos de los cambios demográficos y climáticos que afectarán tanto la oferta como la demanda turística, ya sea para aprovechar las nuevas oportunidades o minimizar los riesgos.

Para futuras investigaciones se sugiere replicar este tipo de trabajos en diferentes destinos considerando los contextos económicos, sociales y culturales a fin de verificar la consistencia de los hallazgos. Así también, se observa la necesidad de vigilar longitudinalmente la evolución de los atributos que generan satisfacción al turista, de modo que se detecten patrones de conducta incorporando las dimensiones ecológicas, demográficas, culturales, de personalidad de los turistas y sus familias, a fin de identificar qué atributos explican la sostenibilidad de un destino turístico y cómo estos motivan la elección y repetición de la visita.

\section{REFERENCIAS}

Alegre, J. y J. Garau, The factor structure of tourist satisfaction at sun and sand destinations. Journal of Travel Research, 50(1), 78-86 (2011)

Alegre, J., M. Cladera, y M. Sard, Analysing the influence of tourist motivations on tourist expenditure at a sun-and-sand destination. doi: 10.5367/te.2011.0063 Tourism Economics, 17(4), 813-832 (2011)

Alén, E., N. Losada, y T. Domínguez, The Impact of Ageing on the Tourism Industry: An Approach to the Senior Tourist Profile. doi: 10.1007/s11205-015-0966-x Social Indicators Research, 127, $303-322$ (2016)

Assaker, G. y R. Hallak, European travelers' return likelihood and satisfaction with Mediterranean sun-andsand destinations: A Chi-square Automatic Identification Detector-based segmentation approach. Journal of Vacation Marketing, 18(2), 105-120 (2012)

Barros, C. y A. Assaf, Analysing tourism return intention to an urban destination. Journal of Hospitality and Tourism Research, 36, 216-231 (2012)

Dogru, T., U. Bulut, y E. Sirakaya, Theory of vulnerability and remarkable resilience of tourism demand to climate change: Evidence from the Mediterranean Basin. Tourism Analysis, 21, 645-660 (2016)

\section{Eyssautier M., Metodología de la investigación. México D.F.: Editorial Thomson (2006)}

Foghagen, C., The blooming paradise: algae blooms, environmental change and tourism. European Journal of Tourism Research, 7, 79-91 (2014) 
Garcia, J., C. Juaneda, J. Raya, y F. Sastre, A study of traveller decision-making determinants: prioritizing destination or travel mode? doi: 10.5367/te.2015.0517 Tourism Economics, 21(6), 1149-1167 (2015)

Hair, J.F., M.W. Celsi, A.H. Money, P. Samouel, M.J., Page Essentials of Business Research Methods Second Edition, M. E.Sharpe Armonk, New York, London, England (2011)

Hampson, D. y P. McGoldrick, A typology of adaptive shopping patterns in recession. Journal of Business Research, 66, 831-838 (2013)

Herington, C., B. Merrilees y H. Wilkins, Preferences for destination attributes: Differences between short and long breaks. doi:10.1177/1356766712463718 Journal of Vacation Marketing, 19(2), 149-163 (2013)

Hernández, R., C. Fernández y P. Baptista, Metodología de la Investigación. México D. F.: Mc Graw-Hill (2006)

INEC, Instituto Nacional de Estadísticas y Censos, Ceso de población y vivienda, (en línea): https://goo.gl/nEMjBv Acceso: 6 de junio de (2017)

Kursah, M. A Quantitative methodology for assessing sustainable tourism potentials. doi: 10.3727/154427217X14866652018901 Tourism Review International, 21(1), 63-80 (2017)

Lai, W.H. y N. Vinh, An application of AHP approach to investigate tourism promotional effectiveness. Tourism and Hospitality Management, 19(1), 1-22 (2013)

Li, H., H. Song, y L. Li, A Dynamic Panel Data Analysis of Climate and Tourism Demand: Additional Evidence. Journal of Travel Research, 56(2), 158-171 (2017)

Lind, D., W. Marchal, y S. Wathen, Estadística aplicada a los negocios y la economía. México D.F.: McGrawHill / Interamericana Editores (2012)

López-Sánchez, Y. y I. Pulido-Fernández, Factors influencing the willingness to pay for sustainable tourism: a case of mass tourism destinations. doi: 10.1080/13504509.2016.1203372 International Journal of Sustainable Development and World Ecology, 24(3), 262-275 (2017)

Luka, M. Decision making in tourism: The choice of city tours in terms of socio-economic status and willingness to pay. Acta Prosperitatis 3, 73-83 (2012)

MINTUR, Ministerio de Turismo, Ecuador, (en línea): http://www.turismo.gob.ec/ Acceso: 10 de marzo de (2017)

Nejati, M., B. Mohamed y S. Omar, The Influence of Perceived Environmental Impacts of Tourism on the Perceived Importance of Sustainable Tourism. E-Review of Tourism Research, 12(1/2), 99-114 (2015)

Prebensen, N., K. Skallerud, y J. Chen, Tourism motivation with sun and sand destinations: Satisfaction and the wom-effect. Journal of Travel and Tourism Marketing, 27(8), 858-873 (2010)

Rodríguez Molina, M. Á., D. M. Frías-Jamilena, y J. A. Castañeda-García, The moderating role of past experience in the formation of a tourist destination's image and in tourists' behavioural intentions. Current issues in tourism, 16(2), 107-127 (2013)

Saverimuttu, V. y M. Varua, Climate variability in the Origin Countries as a "Push" Factor on Tourist Arrivals in the Philippines. Asia Pacific Journal of Tourism Research, 19(7), 846-857 (2014)

Scott, D., S. Gössling, y M. Hall, International tourism and climate change. doi: 10.1002/wcc.165 WIREs Climate Change, 3, 213-232 (2012)

Sheldon, P. y L. Dwyer, The global financial crisis and tourism. Selected papers form the 20th anniversary conference of the International Academy for the Study of Tourism, Mallorca, Spain, June 2009. Journal of Travel Research, 3-45 (2010)

Song, H., L. Dwyer, G. Li, y Z. Cao, Tourism economics research: A review and assessment. Annals of tourism research, 39(3), 1653-1682 (2012) 
Stylos, N., C. A. Vassiliadis, V. Bellou, y A. Andronikidis, Destination images, holistic images and personal normative beliefs: Predictors of intention to revisit a destination. Tourism Management, 53, 40-60 (2016)

Sung, Y.K., K. C. Chang, y Y.F. Sung, Market Segmentation of International Tourists Based on Motivation to Travel: A Case Study of Taiwan. Asia Pacific Journal of Tourism Research, 1-21 (2015)

Tugcu, C. T. Tourism and economic growth nexus revisited: A panel causality analysis for the case of the Mediterranean Region. Tourism Management, 42, 207-212 (2014)

UNWTO. United Nations World Tourism Organization. UNWTO technical manual: collection of tourism expenditure statistics. (en línea) https://goo.gl/dwDuZd (1995) Acceso: 6 de junio de (2017)

Webster, C. y S. Ivanov, Transforming competitiveness into economic benefits: Does tourism stimulate economic growth in more competitive destinations? Tourism Management, 40, 137-140 (2014)

Wilson, J. y Becken, S. Perceived deficiencies in the provision of climate and weather information for tourism: A New Zealand media analysis. New Zealand Geographer, 67, 148-160 (2011) 